\title{
Cardiac sarcoidosis: a case report
}

Rathnaweera RHAI*

Senior lecturer, Department of Forensic Medicine, Faculty of Medicine, Karapitiya, Galle, Sri Lanka

\begin{abstract}
Sarcoidosis is a granulomatous disease of unknown etiology, which can affect people of all racial and ethnic groups and can occur at all ages. Currently, sarcoidosis is considered as a worldwide disease with different prevalence rates. Arrhythmias are the leading cause of sudden death among patients diagnosed with cardiac sarcoidosis. In this case, the deceased was a 51-year-old male, a foreigner, with a history of type II diabetes and hypertension and was on treatment for 12 years. He was found unresponsive in a chair at his residence by his son. Autopsy revealed an enlarged heart (heart weight $543 \mathrm{~g}$, 95th percentile for height is $387 \mathrm{~g}$; 95th percentile for weight is $474 \mathrm{~g}$ ). The pericardial sac is intact. There are no adhesions and no collections of fluid or blood. Epicardium was unremarkable. No acute ischaemic changes were seen in the myocardium. The coronary arteries were normal in configuration with a right dominant circulation. Histopathology of all organs were performed. Sections of myocardium showed varying degrees of replacement fibrosis with an inflammatory infiltrate comprising epithelioid histiocytes, giant cells and occasional non-necrotizing granulomata. There were no acute ischaemic changes. Toxicology screening was negative for common poisons and substance of abuse. These findings were in keeping with cardiac sarcoidosis and concluded it as cause of death. This was a rare case of sarcoidosis where the diagnosis was made with the aid of thorough histopathology examination. The importance of a thorough knowledge on specific cardiac conditions that can cause sudden death is highlighted here.
\end{abstract}

Keywords: Arrhythmia, Sudden cardiac death, Histopathology

Received: 27 April 2020, Revised version accepted: 27 June 2020, Published: 30 June 2020. *Corresponding author: Rathnaweera RHAI, $\triangle$ Email: ajithrathnaweera@gmail.com (D) https://orcid.org/0000-0002-7418-2981

Cite this article as: Rathnaweera RHAI. Cardiac sarcoidosis: a case report. Medico-Legal Journal of Sri Lanka, 2020;8(1): 23-26. DOI: http://doi.org/10.4038/mljsl.v8i1.7406

Copyright: @ 2019 with the Medico-legal Journal of Sri Lanka.

This is an open-access article distributed under the terms of the Creative Commons Attribution 4.0 International License, which permits unrestricted use, distribution and reproduction in any medium provided the original author and source are credited.

\section{Introduction}

Sarcoidosis is a granulomatous disease of unknown etiology, which can affect people of all racial and ethnic groups and can occur at all ages. ${ }^{[1]}$ Environmental, occupational, and infectious causes have been hypothesized as possible triggers. It is believed that a principal triggering event leads to granuloma formation, which can then either resolve or progress to fibrosis. ${ }^{[2]}$ Jonathon Hutchinson, a prominent dermatologic surgeon, first described this clinicopathologic entity among his patients' way back in the mid-19th century. ${ }^{[3]}$ Currently, sarcoidosis is considered as a worldwide disease with different prevalence rates. In the United States and Europe, the annual incidence has been estimated at 5 to 40 cases per 100000 persons. ${ }^{[4]}$ Incidence is 3 to 20 per 100000 for whites and 35.5 to 80 per 100 000 for blacks. ${ }^{[5]}$

The lungs are the commonly affected organ. However, other organs such as heart, liver, spleen, skin, eye, parotid gland or any other organ or tissue can get affected with this condition. ${ }^{[1]}$ It is estimated that in approximately $5 \%$ of the patients diagnosed with sarcoidosis, clinically identifiable cardiac involvement can be seen. ${ }^{[1]}$ Autopsy studies have estimated the prevalence of cardiac involvement in at least $25 \%$ of patients with sarcoidosis in the United States where as it is as high as $58 \%$ in Japanese population. ${ }^{[6]}$ When there is cardiac involvement, the prognosis is not favourable. According to some authors, cardiac sarcoidosis (CS) presented a worse outcome compared to dilated cardiomyopathy. ${ }^{[7]}$

In patients with $\mathrm{CS}$, various forms of arrhythmias have been reported including atrial and ventricular ones. Out of that, atrioventricular (AV) block is the most common variety followed by ventricular tachyarrhythmias (VT) and ventricular fibrillation (VF). ${ }^{[8]}$ Arrhythmias are the leading cause of sudden death among patients diagnosed with cardiac sarcoidosis. Involvement of the basal interventricular septum due to fibrous / scar tissue formation and formation of sarcoid granulomas are supposed to be the cause for most of these arrhythmias. ${ }^{[8]}$ 


\section{Case report}

The deceased was a 51-year-old male, a foreigner, with a history of type II diabetes and hypertension and was on treatment for 12 years. He was found unresponsive in a chair at his residence by his son. No suspicious circumstances were identified. He was immediately brought to a nearby hospital where he was found to be in cardiac arrest. Cardiopulmonary resuscitation was attempted for about 20 minutes and later he was pronounced dead.

At the autopsy, the pericardial sac was found to be intact and free of any collection of fluids. The heart weight was $543 \mathrm{~g}$. The right ventricular cavity diameter was $35 \mathrm{~mm}$ and the left ventricular cavity diameter was $45 \mathrm{~mm}$. The right ventricular wall thickness was $5 \mathrm{~mm}$ and the left ventricular free wall thickness was $20 \mathrm{~mm}$. The interventricular septum measured $20 \mathrm{~mm}$ in thickness and was intact. The endocardium appeared unremarkable. The cardiac valves were normal in configuration. The coronary arteries were normal in configuration with a right dominant circulation. The right and left coronary ostia were patent and no significant atherosclerotic stenosis of the coronaries were seen. The aorta showed a smooth intima with no evidence of dissection or aneurysm. The carotid arteries were examined to their bifurcation and showed no significant stenosis. The pleural cavity was free from any collection of fluid. The left lung weighed $410 \mathrm{~g}$ and the right lung weighed $447 \mathrm{~g}$. On sectioning, the parenchyma bilaterally was mildly congested. The brain was normal in configuration. Serial coronal sections through the cerebral hemispheres did not show any abnormality of the cerebral cortex, underlying white matter or basal ganglia. The liver weighed $3102 \mathrm{~g}$ with an intact capsule. The parenchyma was soft with a uniform yellow brown appearance in keeping with steatosis. The left kidney weighed $175 \mathrm{~g}$. and the right $210 \mathrm{~g}$ and the both kidneys were macroscopically unremarkable. The spleen weighed $445 \mathrm{~g}$ and was unremarkable.

Histopathology of all organs were performed. Sections of myocardium showed varying degrees of replacement fibrosis with an inflammatory infiltrate comprising epithelioid histiocytes, giant cells and occasional non-necrotizing granulomata (Figure 1, Figure 2, Figure 3).

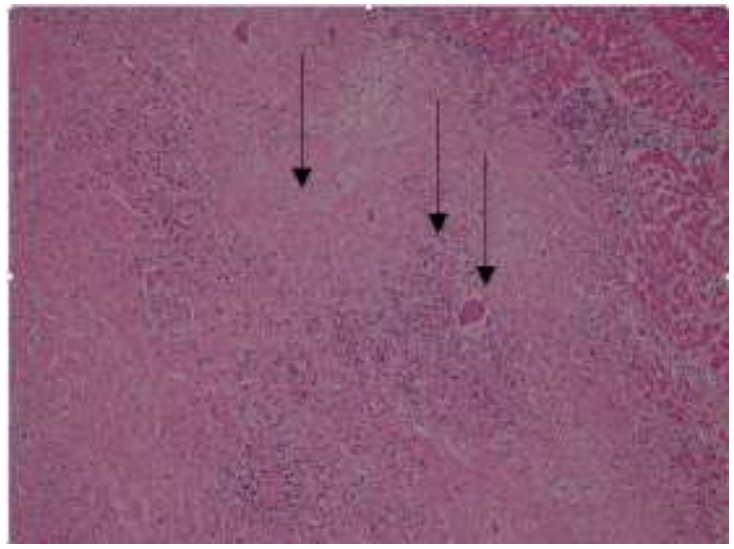

Figure $1-\mathrm{H}$ \& E stain Low power x 10 Replacement fibrosis, non-caseating granulomata, giant cells

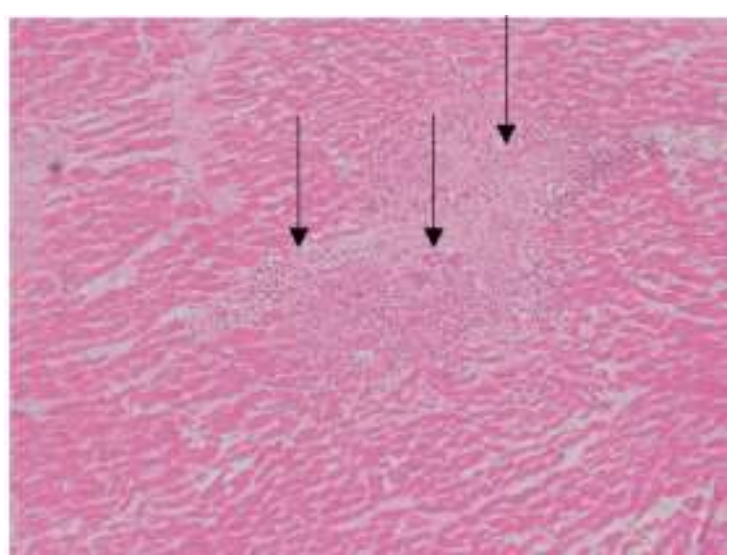

Figure 2 - H \& E stain Medium power x 20 Replacement fibrosis with an inflammatory infiltrate comprising epithelioid histiocytes, giant cells

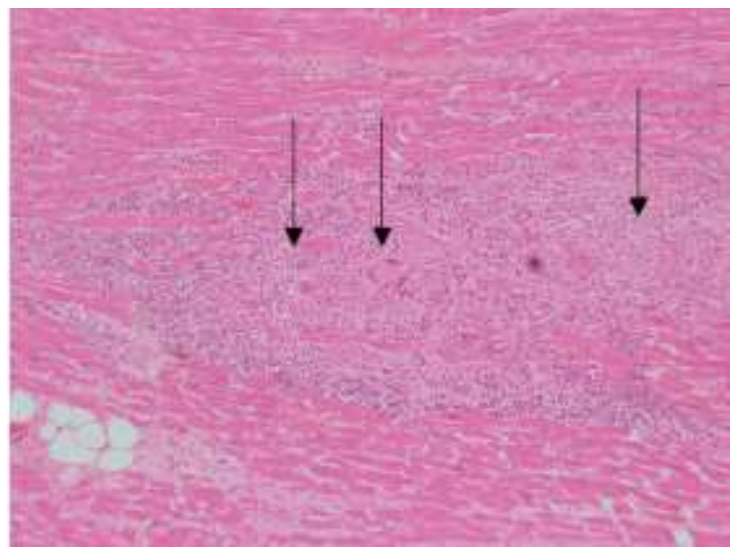

Figure 3 - H \& E stain Medium power x 20 Replacement fibrosis with an inflammatory infiltrate comprising epithelioid histiocytes, giant cells

There were no acute ischaemic changes. These findings were in keeping with cardiac sarcoidosis. Sections of lung tissue did not reveal any granulomata. There were patchy alveolar macrophages throughout all lung lobes. The bronchi showed prominent basement membrane material with some smooth muscle hypertrophy and 
patchy chronic inflammatory infiltrates including occasional eosinophils. The hepatic architecture was within normal limits. The portal tracts were prominent with a mild lymphocytic infiltrate surrounding them. There were no granulomata. There was moderate steatosis with focal glycogenation of the hepatocyte nuclei. The renal tissue showed evidence of benign nephrosclerosis and moderate intimal proliferation of the larger vessels. There was no significant inflammatory infiltrate. The glomeruli were within normal limits. The cause of death was given as cardiac sarcoidosis and circumstance was concluded as natural after an inquest.

\section{Discussion}

Sarcoidosis is said to be slightly common in females than males. ${ }^{[7]}$ However, myocardial involvement does not show any gender preference according to the current data. ${ }^{[8]}$ The disease most commonly presents in adults younger than 40 years with 20 to 40-year age group is the most vulnerable population. ${ }^{[7,}$ In this case, the deceased was a 51year-old male.

Patients with CS can present with a wide range of signs and symptoms beginning with minor Electrocardiograph changes to sudden death. The underlying pathology and its extension determines the initial presenting symptom and this could include palpitations, dyspnea, syncope, dizziness, chest pain, orthopnoea or peripheral oedema. ${ }^{[1,7,9]}$ Sudden cardiac death, ventricular arrhythmia, atrial arrhythmia, other conduction system disease, congestive heart failure, papillary muscle dysfunction, mitral insufficiency, myocardial infarction, ventricular aneurysm, cor pulmonale, and pericardial effusion can all be presentations of cardiac sarcoidosis. ${ }^{[7,9]}$ In this case, the presenting symptom was sudden cardiac arrest.

Clinical diagnosis of CS is not straight forward and sometimes can get misdiagnosed as dilated cardiomyopathy, arrhythmogenic right ventricular cardiomyopathy or as an idiopathic ventricular aneurysm. This is mainly due to nonspecific ECG and echocardiographic findings. ${ }^{[1,2,7,9]}$ Sarcoid granulomas may involve pericardium, myocardium or endocardium, however, myocardium remains the most frequently involved tissue. Myocardium of the left ventricular free wall is the most common location of sarcoid granulomas, followed by the septum, right ventricle, and atria. Cardiac conduction system is also a commonly affected site. ${ }^{[1,7,8]}$ A definitive diagnosis of cardiac sarcoidosis requires the histologic examination of tissue specimens obtained by cardiac biopsy. However, sensitivity can be as low as $(<25 \%)$ with the usual right ventricular biopsies. This is because of the sampling error because of the patchy or focal involvement of the myocardium..$^{[1,2]}$

Because of the segmental involvement of the myocardium, histopathology diagnosis may become difficult even at the autopsy. Accurate sampling techniques are required in every suspected case to successfully identify this disease condition. Also, a thorough understanding of the disease process and a sound knowledge on histopathology changes are very essential when making a proper diagnosis of cardiac sarcoidosis.

The characteristic lesion of sarcoidosis is a discrete, compact, non-caseating epithelioid cell granuloma without a great deal of lymphocytic inflammation. Langhans type giant cells with or without Schaumann bodies and asteroid bodies; and the presence of patchy fibrosis can all help to make the diagnosis. $^{[2]}$

The primary histologic differential diagnosis for cardiac sarcoidosis includes giant cell myocarditis, idiopathic (nonspecific) granulomatous myocarditis, tuberculous myocarditis, fungal myocarditis, and Whipple disease. ${ }^{[1,2]}$ Although there are no pathognomonic features to differentiate sarcoidal granuloma from another cause, tight, naked, nonnecrotizing, and epithelioid granulomas usually favors sarcoidosis.

There is an ongoing debate over differentiating features of cardiac sarcoidosis and granulomatous myocarditis. Some people believe that granulomatous myocarditis is a part of the spectrum of pathology in cardiac sarcoidosis while others believe that these are two different entities. The multicenter observational study by Okura et al. on clinical and histopathologic comparison of cardiac sarcoidosis and giant cell myocarditis revealed some clear histopathological differences. ${ }^{[10]}$ Eosinophils, myocyte damage, and foci of lymphocytic myocarditis were frequently observed in idiopathic giant cell myocarditis, while granulomas and fibrosis were more frequent in sarcoidosis. ${ }^{[10]}$ Similar observations were made by other authors. In a case report by Bogabathina et al, non-caseating granulomatous inflammation with many multinucleated giant cells, histiocytes, and mild chronic inflammation was observed in the cardiac biopsies. ${ }^{[11]}$ Sarcoidosis was favored over idiopathic giant cell myocarditis because of the presence of well-formed granulomas and granulomatous inflammation. ${ }^{[11]}$

On the other hand, identification of organisms within the granuloma effectively rules out sarcoidosis. ${ }^{[1,2,9]}$ The macrophages within sarcoid granulomas tend to become epithelioid and form multinucleated giant cells. The multinucleated giant 
cells are mostly foreign body type initially and in later stages they are mostly Langhans type. Some of these giant cells may contain cytoplasmic inclusions, particularly Schaumann bodies or asteroid bodies. ${ }^{[2,9,12,13]}$

In this case, the post mortem examination revealed an enlarged heart with a predominantly subendocardial distribution of a non-necrotizing, noncaseating granulomatous infiltrate associated with varying degrees of fibrosis. The granulomas were well formed. There were areas of prominent fibrosis. No significant myocyte necrosis was seen. Occasional asteroid bodies were seen within the giant cells and there were varying types of giant cells including Langhans and foreign body types. All these features favor a diagnosis of cardiac sarcoidosis.

In patients with CS, various arrhythmias have been reported including atrial and ventricular ones. ${ }^{[14,15]}$ In this case, the most likely mechanism of death is a cardiac arrhythmia, especially considering the sudden nature of the cardiac arrest. His heart enlargement (cardiomegaly) was probably due to a sarcoid-related cardiomyopathy.

\section{Conclusions}

This was a rare case of cardiac sarcoidosis where the diagnosis was made with the aid of thorough histopathology examination. The importance of a thorough knowledge on specific cardiac conditions that can cause sudden death is highlighted here for the better understanding of disease processes.

\section{Disclosure statement}

Conflicts of interests: The authors declare that they have no conflicts of interests.

\section{Funding: None}

\section{References}

1. Birnie DH, Nery PB, Ha AC, Beanlands SB, Cardiac Sarcoidosis. JACC, 2016; 8(4): 411-21

2. Sekhri V, Sanal S, Delorenzo LJ, Aronow WS, Maguire GP. Cardiac sarcoidosis: a comprehensive review. Arch Med Sci, 2011; 7(4): 546-54
3. Hutchinson J. Cases of Mortimer's malady, a form of lupus pernio. Arch Surg. 1898; 9 (1): 307-15

4. Rybicki BA, Major M, Popovich J Jr, et al. Racial differences in sarcoidosis incidence: a 5year study in a health maintenance organization. Am J Epidemiol 1997;145 (3):234-41.

5. Patel AR, Rashedi N. Cardiac Sarcoidosis: A Picture May Be Worth a Thousand Words, But Do We Need More?. J Am Heart Assoc, 2019;8(10): e012715.

6. Matsui $Y$, Iwai $K$, Tachibana $T$, et al. Clinicopathological study on fatal myocardial sarcoidosis. Ann N Y Acad Sci, 1976; 278 (1) : 455-69

7. Lagana SM, Parwani AV, Nichols LC. Cardiac Sarcoidosis: A Pathology-Focused Review. Arch Pathol Lab Med, 2010; 134(1): 1039-46

8. Ipek $\mathrm{E}$, Demirelli $\mathrm{S}$, Ermis $\mathrm{E}$, Inci $\mathrm{S}$. Sarcoidosis and the heart: A review of the literature. Intractable \& Rare Diseases Research, 2015; 4(4):170-80

9. Doughan A, Williams B. Cardiac Sarcoidosis. Heart, 2006;92 (2) :282-88

10. Okura Y, Dec W, Hare JM, Kodama M, Berry GJ et al. A clinical and histopathologic comparison of cardiac sarcoidosis and idiopathic giant cell myocarditis. J Am Coll Cardiol, 2003 Jan;41(2): 322-29.

11. Bogabathina H, Olson $\mathrm{P}$, Rathi VK, Biederman RWW. Cardiac Sarcoidosis or Giant Cell Myocarditis? On Treatment Improvement of Fulminant Myocarditis as Demonstrated by Cardiovascular Magnetic Resonance Imaging. Case reports in Cardiology, 2012 Jan; 2012(1):01-05

12. Perry A, Vuitch F. Causes of death in patients with sarcoidosis: a morphologic study of 38 autopsies with clinicopathologic correlations. Arch Pathol Lab Med,1995;119(2): 167-72

13. Hulten E, Aslam S, Osborne M, Abbasi, S, Bittencourt MS, Blankestein R. Cardiac sarcoidosis - state of the art review. Cardiovasc Diagn Ther, 2016 Feb; 6(1): 50-63.

14. Kim JS, Judson MA, Donnino R, et al. Cardiac sarcoidosis. Am Heart J, 2009;157(1):9-21

15. Kusano KF, Satomi K. Heart, 2015;2(1):1-7 doi:10.1136/heartjnl-2015-307877 